\title{
Combined caesarian section, mitral valve replacement and tricuspid valve repair for infective endocarditis: case report and management considerations
}

\author{
Galya Atanasova* \\ Medical University, Pleven, 5800 Pleven, Tzar Samuil Str, Bulgaria
}

\begin{abstract}
A 35 year old woman in her third trimester of pregnancy presented with fever and left flank pain. The presumptive diagnosis was pyelonephritis. Further evaluation demonstrated a cardiac murmur. Echocardiography showed a mass on the anterior leaflet of the mitral valve (MV). A history of recent dental surgery was elicited. Cardiology consultation was obtained and empiric intravenous antibiotic therapy (i.e., Cefuroxime) initiated. All cultures (i.e., Blood and urine) were negative. Despite medical and antimicrobial management, she developed congestive heart failure. She was transferred to the university hospital where she was given diuretics and additional antibiotics. Repeat echocardiography showed progression in the size $(1.7 \times 2.2 \mathrm{~cm})$ of the MV mass, now considered vegetation. Fetal assessment was normal. Cardiothoracic surgery consultation was obtained and the decision was made to proceed with surgery. A Caesarian section followed by MV Replacement with a mechanical prosthesis and Tricuspid Valve Repair (i.e., DeVega) were successfully performed. Adult and neonatal monitoring was utilized intraoperatively. Postoperatively, the mother was treated with extended antibiotic therapy and anticoagulation with warfarin; the newborns were managed in the neonatal ICU without complication. The mother and children were subsequently discharged in stable condition.
\end{abstract}

\section{Introduction}

Cardiovascular disease in pregnancy poses a unique problem because two lives are involved-mother and fetus. Although medical management of this state is challenging, considerable improvement in care has been observed in the past several decades. However, when surgery is necessary, the complexity of the situation becomes more problematic. Depending upon the nature of the surgical intervention, the risk to both mother and fetus can be significant. Although maternal death is less common now than in the past, the risk to both mother and fetus are not insignificant. For example, in a systematic review of patients that underwent a non-obstetric surgical intervention during pregnancy (i.e., 12,452 patients), the incidence of fetal loss was not trivial, particularly when the surgery occurred in the first trimester [1]. The stress of surgery, the hemodynamic consequences during surgery, the medications and anesthetics needed during the surgery, and the combined adult and perinatal monitoring are all factors that need to be taken into account in pregnant patients undergoing operative intervention. Perhaps the most challenging scenario is the pregnant woman in need of heart surgery-a state that adds another layer of risk: the heart-lung machine. The purpose of this report is to describe a third-trimester woman carrying twins who developed infective endocarditis (IE) of the mitral valve (MV) that necessitated surgical intervention. The perioperative issues are described in the context of pre-, intra-, and post-operative factors.

\section{Case report}

A 35-year-old woman in the third trimester of her twin pregnancy presented with 102.2 F fever and left flank pain, frequent urination, lower extremity swelling, palpitations, and dyspnea/orthopnea for several weeks. She was admitted to the Clinic of Pathologic Pregnancy for further evaluation. The history was significant for a recent dental procedure requiring tooth reconstruction. The mother's examination was positive for high-grade fever, tachycardia, left flank pain, and a cardiac murmur. An echocardiography showed a $2.2 \times 1.7 \mathrm{~cm}$ vegetation on the anterior leaflet of the mitral valve along with $3+$ mitral regurgitation. Empiric antibiotic therapy with Cefuroxime was initiated. Blood and urine cultures were negative. The fetal examination with serial scans was normal. The presumptive diagnosis was pyelonephritis along with MV endocarditis. The plan was medical management with close observation and follow-up echocardiography.

The patient continued intravenous Cefuroxime therapy for seven days during which time she became afebrile. However, she went on to develop signs and symptoms of pulmonary congestion. She was transferred to the Cardiology Clinic at the University Hospital. The examination demonstrated physical deterioration: she was weak, listless, and dyspneic with pale mucous membranes. Her lung sounds were positive for rales and there was a loud systolic cardiac murmur. In addition, there were splinter hemorrhages, petechiae, and exanthema. She was tachycardic. Laboratory values showed mild anemia ( $\mathrm{Hgb}$ $10.5 \mathrm{G} / \mathrm{DL}$ ), mild leukocytosis (WBC $12.2 \mathrm{~K} / \mathrm{UL}$ ), elevated C-Reactive Protein (32.46 MG/L), and proteinuria. All microbiologic cultures were negative. Repeat echocardiography revealed progression in size and mobility of the MV vegetation (Figures 1 and 2) In addition, there was severe mitral regurgitation with left atrial enlargement and a small pericardial effusion. The left ventricle was normal in size with

Correspondence to: Galya Atanasova, Medical University, Pleven, 5800 Pleven, Tzar Samuil Str, Bulgaria, Tel: +359889 202 647; Fax: +359889 202 647; E-mail: maa_05@abv.bg

Received: June 19, 2016; Accepted: July 07, 2016; Published: July 11, 2016 


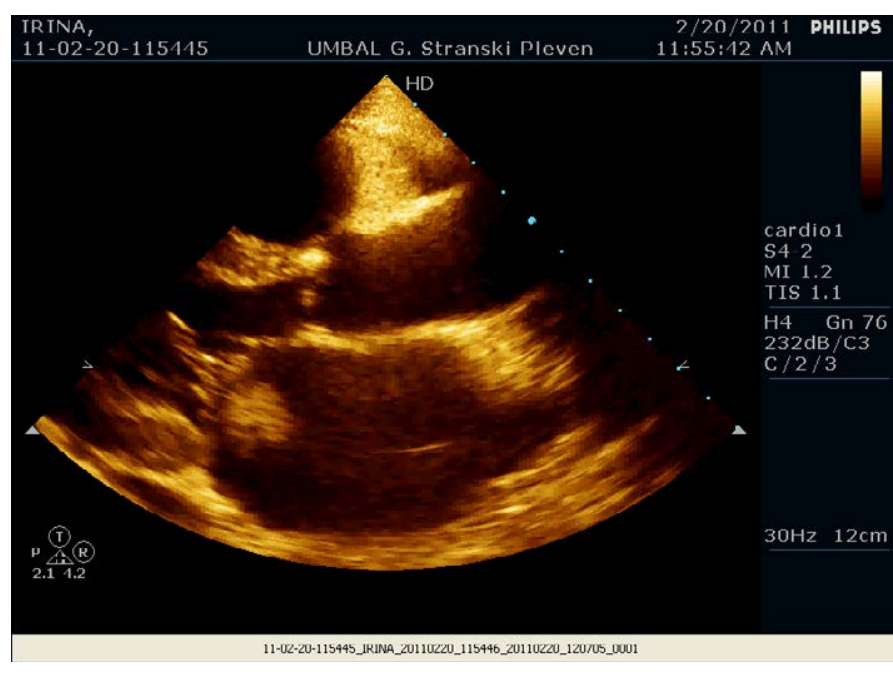

Figure 1. Echocardiogram showing mitral valve vegetation prolapsing into the left atrium.

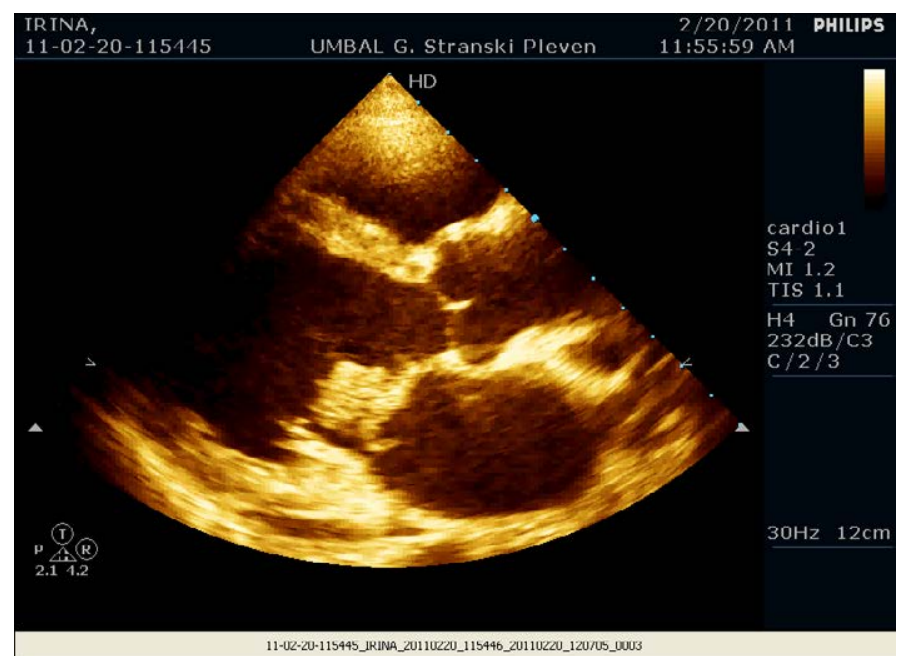

Figure 2. Echocardiogram showing large mitral valve vegetation prolapsing into the left ventricle.

a hyperdynamic quality. The aortic valve was structurally normal and its function was intact. There was mild right ventricular and right atrial dilatation with tricuspid regurgitation; Doppler assessment showed elevated systolic pulmonary artery pressure. She was treated with intravenous diuretic therapy and digoxin. In addition, her antibiotic coverage was broadened with gentamycin and Ceftriaxone. Her condition improved during the next seven days after which she was transferred to the Department of Cardiothoracic Surgery.

The patient was prepared for surgery with both maternal and fetal monitoring. A Caesarian Section was performed without incident. Following the delivery, preparation was made for open heart surgery. Establishment of cardiopulmonary bypass (CPB), a Mitral Valve Replacement (MVR) using a $31 \mathrm{~mm}$ Carbomedics (Sorin S.p.A., Milan, Italy) mechanical prosthesis and a DeVega Tricuspid Valve annuloplasty were performed. Postoperatively, the mother and neonates were managed in their respective intensive care units. There were no major postoperative complications. The mother was discharged on the twenty-fourth hospital day on anticoagulation (i.e., Warfarin), angiotensin converting enzyme inhibitor, furosemide, digoxin, and antibiotics (i.e., Cefuroxime and Ketoconazole).
The patient was re-admitted a month later with fever and a diffuse rash. Laboratory values were within normal limits with only a mild leukocytosis (i.e., WBC $11 \mathrm{~K} / \mathrm{UL}$ ). The vital signs were stable except for a mild tachycardia. Clinically, she appeared markedly improved. Echocardiography demonstrated preserved biventricular function with normal mechanical prosthetic mitral valve and native tricuspid valve function. Allergy consultation was obtained and the diagnosis was medication reaction. She was treated with steroids and antihistamines with a favorable response.

\section{Discussion}

Cardiovascular disease in pregnancy is a challenging problem since two lives are at risk. The need for cardiovascular surgery during pregnancy is even more problematic since the procedures and medications needed to conduct the operation can potentially harm the mother and/or the fetus, particularly during the first trimester of gestation where fetal loss is highest. Unfortunately, female gender, youth, and pregnancy do not afford protection against some of the same cardiovascular maladies that affect older patients. Infectious endocarditis is no exception.

Although uncommon, the incidence of IE in pregnancy has been reported to be $0.006 \%$ ( 1 per 100000 pregnancies) with an incidence of $0.5 \%$ in patients with known valvular or congenital heart disease $[2,3]$. Despite its rarity, IE during pregnancy has been a problem for decades with various outcomes based upon experience and available resources. Although technological advances have been made along with improvements in operative technique, critical care, and neonatal monitoring, the fundamental goal of IE cases during pregnancy is universally unchanged: mother and child survival. Historically, the disease has the propensity to be fatal. As reported in a MEDLINE review from 1965 to 2003, the calculated maternal and fetal mortality rates were $22.1 \%$ and $14.7 \%$ respectively [4]. In the modern era of heart surgery, significant progress has been made in terms of understanding the factors associated with maternal and fetal survival [5].

Efforts to reduce risk have been successful as improvements in critical care for both adults and neonates have been realized. As in any case of IE-with or without associated pregnancy-- there are established guidelines in management which are updated regularly. According to the American Heart Association (AHA) and the American College of Cardiology (ACC), medical therapy with antibiotics is the first order of therapy. The indications for surgery are reserved for the following states: moderate-to-severe congestive heart failure, valve dysfunction, perivalvular or myocardial abscess formation, the presence of an unstable valve that is becoming detached from the valve ring, more than one embolic episode with persistent vegetations observed on transtracheal echocardiogram, or the presence of vegetations larger than $1 \mathrm{~cm}$ in diameter [6,7]. Similar guidelines are represented in the European Society of Cardiology (ESC) with a comprehensive table outlining the indications and timing of left-sided native valve endocarditis [8]. As a result of these advanced guidelines, the recognition, treatment, and management of IE in general-and of IE during pregnancy specifically-has improved. However, as helpful as these guidelines are, the surgical details, including anesthesia, perfusion, and intra-operative monitoring need to be equally appreciated.

The experience with cardiac surgery during pregnancy is well represented in the literature, dating back to the earliest years before the advent of the heart-machine [9]. Among the first to report cardiac surgery during pregnancy utilizing extracorporeal circulation was Dr. Cooley and his team from Houston, Texas in which 3 cases 

considerations

were reported in 1965 [10]. The experience of cardiac surgery in the pregnant patient has grown considerably since then, allowing for progress in surgical technique, perfusion and anesthetic management. For example, modern literature supports the following steps during cardiac surgery in the pregnant patient: use of high-flow, highpressure, normothermic bypass for as brief a period as possible [11]. The rationale for this approach is based on the reduction in the fetal heart rate in response to $\mathrm{CPB}$. The bradycardia is thought to be due to hypoperfusion secondary to uterine contractions-this condition can be reversed by increasing the perfusion rate. Specific recommendations for $\mathrm{CPB}$ parameters include: 1) maintaining the pump flow rate $>2.5$ $\mathrm{L} / \mathrm{min} / \mathrm{m}^{2}$ and perfusion pressure $>70 \mathrm{mmHg}$; 2) maintaining the hematocrit $>28 \%$; 3) using normothermic perfusion; 4) using pulsatile flow; and 5) using alpha-stat $\mathrm{pH}$ measurement [12]. These maneuvers have translated into a reduction in both maternal and fetal mortality and morbidity [13]. In addition to these operative maneuvers, equally important surgical decisions need to be made regarding the surgical procedure itself. In the past, the surgical options were limited. Today, the choices are several.

The decisions regarding the operative approach to the diseased valve must take into consideration the option of valve repair or replacement-and if replacement, the choice of a biological or mechanical prosthesis. For obvious reasons, valve repair is preferred in order to avoid the issues related to prosthetic valve durability (i.e., in the case of a biological device) or anticoagulation with warfarin (i.e., in the case of a mechanical device) [14]. In the early years of heart valve surgery, repair was unusual since the techniques for repair were not perfected; similarly, biological prosthetic valve replacement was less common than mechanical because the integrity/durability of the "early" biological prostheses were uncertain. As a result, mechanical prostheses were used more commonly. However, because pregnant patients are young, mechanical prostheses are still utilized with relative frequency-the advantage of long-term durability is weighed against the absolute need for blood thinners that cross the placenta. These same concerns now existed decades ago: postoperative anticoagulation in a pregnant patient with an already existing prosthetic heart valve [15]. Since the use of warfarin in pregnancy is associated with major fetal complications, alternative agents have been considered for the pregnant patient with a mechanical heart valve. The use of low molecular weight heparin, for example, has been studied. While on the surface this drug appears to be an attractive alternative to warfarin, the results, unfortunately, have been somewhat disappointing. In fact, there were significant bleeding and thrombotic complications with one study reporting a $43 \%$ fetal or neonatal adverse event rate $[16,17]$. Thus, the decision at the time of surgery needs to take into account the issue that the pregnant patient with IE is young and may become pregnant again. As such, a valve repair or biological valve replacement would be desirable in order to avoid the complications associated with life-long warfarin therapy (or its alternative) should they be needed. This decision should be made jointly between surgeon and patient, as pointedly described by Guler et al. in a letter to the editor of a Turkish journal [18].

In the current era of biological prosthetic valves in the mitral position, the degeneration rate at 10 years in patients less than 40 years of age is approximately $20 \%$ [19]. Furthermore, the risk for reoperation in the contemporary era is very acceptable. In a recent manuscript by Dr. Chitwood's team from East Carolina University over a 15 year period, the 30-day operative mortality for redo MVR was only $3.0 \%$ [20]. Furthermore, there is emerging experience with transcatheter valve-in-valve cases, including those for degenerative mitral valve prostheses [21]. Thus, in the modern era of cardiovascular medicine and surgery, the statistics of the distant past no longer apply and recent outcomes warrant consideration toward maternal and fetal survival. In addition, the decisions pre-operatively and intra-operatively have profound implications post-operatively to the extent that the decision options should be discussed in advance (if possible) knowing that a) the patient is young, and b) there are more therapies now than existed before.

\section{Summary}

In summary, cardiac surgery in pregnancy has evolved considerably, more so in the past decade than ever before. Despite the risk to mother and fetus, proper recognition and management of the pregnant patient in need of heart surgery can minimize this risk. The case illustration depicts the state-of-the-art as it pertains to a third-trimester woman with endocarditis of the mitral valve in need of surgery. Proper preoperative preparation, intraoperative techniques and monitoring, as well as postoperative care translated into a successful outcome. Other related cases, such as aortic valve sparing and mitral valve repair in a pregnant woman [22], combined ascending aortic dissection and Caesarian section [23], and rheumatic heart disease requiring surgery in pregnant patients [24], have all been reported in this calendar year (2013) with excellent results. Based upon the developments in surgical and interventional technologies and techniques, critical care monitoring and therapies, further improvements in outcome for pregnant patients requiring cardiac intervention is likely to occur.

\section{Acknowledgements}

I wish to express my sincere gratitude to all those who have generously contributed to this case. Thank you for allowing me to use your data.

\section{References}

1. Cohen-Kerem R, Railton C, Oren D (2005) Pregnancy outcome following non-obstetric surgical intervention. Am J Surg 190: 467-473. [Crossref]

2. Avila WS, Rossi EG, Ramires JA, Grinberg M, Bortolotto MR, et al. (2003) Pregnancy in patients with heart disease: experience with 1,000 cases. Clin Cardiol 26: 135-142. [Crossref]

3. Cox SM, Hankins GDV, Leveno KJ, Cunningham FG (1988) Bacterial endocarditis: a serious pregnancy complication. J Reprod Med 33: 671-674. [Crossref]

4. Campuzano K, Roque H, Bolnick A (2003) Bacterial endocarditis complicating pregnancy: case report and systematic review of the literature. Arch gynecol Obstet 268: 251-255 [Crossref]

5. Mahli A, Izdes S, Coskun D (2000) Cardiac operations during pregnancy: review of factors influencing fetal outcome. Ann Thorac Surg 69: 1622-1626. [Crossref]

6. Baddour LM, Wilson WR, Bayer AS (2005) Infective endocarditis: diagnosis, antimicrobial therapy, and management of complications: a statement for healthcare professionals from the Committee on Rheumatic Fever, Endocarditis, and Kawasak Disease, Council on Cardiovascular Disease in the Young, and the Councils on Clinical Cardiology, Stroke, and Cardiovascular Surgery and Anesthesia, American Heart Association: endorsed by the Infectious Diseases Society of America. Circulation 111: e394-434. [Crossref]

7. Nishimura RA, Carabello BA, Faxon DP, et al. (2008) ACC/AHA 2008 guideline update on valvular heart disease: focused update on infective endocarditis: a report of the American College of Cardiology/American Heart Association Task Force on Practice Guidelines: endorsed by the Society of Cardiovascular Anesthesiologists, Society for Cardiovascular Angiography and Interventions, and Society of Thoracic Surgeons. Circulation. Aug 118: 887-896.

8. Habib G, Hoen B, Tornos P (2009) Guidelines on the prevention, diagnosis, and treatment of infective endocarditis (Version 2009).The Task Force on the prevention, diagnosis, and treatment of infective endocarditis of the European Society of Cardiology (ESC). Endorsed by the European Society of Clinical Microbiology and 
Atanasova G (2016) Combined caesarian section, mitral valve replacement and tricuspid valve repair for infective endocarditis: case report and management considerations

Infectious Disease (ESCMID) and by the International Society of Chemotherapy (ISC) for infection and cancer. Eur Heart J 30: 2369-2413.

9. Harken DE, Taylor WJ (1961) Cardiac surgery during pregnancy. Clin Obstet Gynecol 4: 697-709.

10. Jacobs WM, Cooley D, Goen GP(1965) Cardiac surgery with extracorporeal circulation during pregnancy: report of 3 cases. Obstet Gynecol 25: 167-169.

11. Parry AJ, Westaby S (1996) Cardiopulmonary bypass during pregnancy. Ann Thorac Surg 61:1865-1869.

12. Chandrasekhar S, Cook CR, Collard CD (2009) Cardiac surgery in the parturient. Anesth Analg 108: 777-785. [Crossref]

13. Pomini F, Mercogliano D, Cavalletti C (1996) Cardiopulmonary bypass in pregnancy. Ann Thorac Surg 61: 686-690.

14. Sternik L, Zehr KJ, Orszulak TA (2002) The advantage of repair of mitral valve in acute endocarditis. J Heart Valve Dis 11: 91-98. [Crossref]

15. Tejani N (1973) Anticoagulant therapy with cardiac valve prosthesis during pregnancy. Obstet Gynecol 42:785-793

16. Yinon Y, Siu Sc, Warshafsky C (2009) Use of low molecular weight heparin in pregnant women with mechanical heart valves. Am J Cardiol 104: 1259-1263. [Crossref]

17. McLintock C, McCowan LM, North RA (2009) Maternal complications and pregnancy outcome in women with mechanical prosthetic heart valves treated with enoxaparin BJOG 116: 1585-1592. [Crossref]

18. Guler A, Sahin MA, Kucukarsian N (2010) A case of infective endocarditis, during pregnancy: should we keep the fetus? Anadolu Kardiyol Derg 10: 291-296. [Crossref]

19. Jamieson WR, Ling H, Burr LH (1998) Carpentier-Edwards supraannular bioprosthesis evaluation over 15 years. Ann Thorac Surg 66: S49-S52. [Crossref]

20. Arcidi JM Jr, Rodriguez E, Elbeery JR (2012) Fifteen-year experience with minimally invasive approach for reoperations involving the mitral valve. $J$ Thorac Cardiovasc Surg 143: 1062-1068. [Crossref]

21. Seiffert M, Conradi L, Baldus S (2012) Transcatheter mitral valve-in-valve implantation in patients with degenerated bioprostheses. JACC Cardiovasc Interv 5 : 341-349. [Crossref]

22. Tang GH, Malekan R, Lansman SL (2013) Aortic valve-sparing reimplantation and mitral repair in a pregnant, second trimester Marfan patient: surgical decision. Ann Thorac Surg 95: 701-703. [Crossref]

23. Lichtman AD, Kjaer K (2013) Combined Caesarian delivery and repair of acute ascending and aortic arch dissection at 32 weeks of pregnancy. $J$ Cardiothorac Vasc Anesth 27: 731-734

24. Birincioglu CL, Unal EU, Celik IH (2013) Surgery for rheumatic valve disease in pregnancy: What about the newborn? Heart Lung Circ S1443-9506 (Epub ahead of print). [Crossref]

Copyright: (C2016 Atanasova G. This is an open-access article distributed under the terms of the Creative Commons Attribution License, which permits unrestricted use, distribution, and reproduction in any medium, provided the original author and source are credited. 